\title{
Psychological Interventions in Improving Positive Body Image in Adolescents: A Systematic Review
}

\author{
Retno Puji Astuti ${ }^{1}$, Rizki Fitryasari $^{1 *}$, Hendy Muagiri Margono \\ ${ }^{I}$ Faculty Of Nursing, Airlangga University, Indonesia \\ ${ }^{2}$ Faculty Of Medicine, Airlangga University, Indonesia \\ *rizki-fpk@fkp.unair.ac.id
}

\begin{abstract}
Body image is an important issue for adolescents. This is related to the surrounding appearance that is considered attractive by most people where beautiful people are those who have a tall body and white skin. This is influenced by a fast-developing lifestyle so adolescents who are in the phase of looking for self-identity want to look their best by current appearance criteria. The purpose of this Systematic review is to analyze the effect of psychological interventions on adolescent's body image. Systematic review accessed five electronic databases (Scopus, MEDLINE, CINAHL, Science Direct, and ProQuest). The search was carried out from April to June. Assess the quality of the article used The Center for review and Dissemination and the Joanna Briggs Institute Guideline and the Prisma checklist as a guide in making this review. Title, abstract, full-text, and methodology were assessed for study eligibility. 25 articles were analyzed. Most of the psychological interventions provided offline were 16 studies and the rest were through indirect intervention methods. The research design consisted of 17 randomized control trials and 8 studies using quasi-experimental. The number of samples varied from 22 respondents to 2481 respondents. Two types of psychological interventions can be used as alternatives to enhance and maintain a positive body image: direct psychological intervention and indirect psychological intervention using technology. Psychological intervention is very important in enhancing and maintaining a positive body image.
\end{abstract}

Keywords : Adolescents, Body Image, Psychological Intervention 


\section{STRADA Jurnal Ilmiah Kesehatan}

DOI: $10.30994 /$ sjik.v9i2.385

ISSN: 2252-3847 (print); 2614-350X (online)

Vol.9 No.2 November 2020 Page.772-789

\section{BACKGROUND}

Attention to the image of a person's body is very strong in adolescents aged 12-18 years, both young women and young men. They try to get their appearance in various ways to change their unwanted appearance, but this does not necessarily guarantee adolescent satisfaction with their bodies (Santrock, 2007). Appearance is very important for a woman. Therefore, women need to maintain an appearance that can attract the attention of others.

Appearance is very important for a woman. The standard of beauty in society for an ideal woman is someone who has a slim body (Mumford \& Choudry, 2000), which causes many women to feel dissatisfied with their current weight and body shape (Wong, 2008). Therefore, many women make every effort to achieve ideal standards, especially body shape. Attention to a person's body image is very strong in adolescents aged 12-18 years, both young women and young men. They try to get a display in various ways to change the appearance that is not desired (Santrock, 2007).

The population of teenagers in the world is around 1.2 billion or $18 \%$ of the world's population (WHO, 2017). Meanwhile, in Indonesia 25\% (66, 3 million) of the population 10-24 years old adolescents of the total population of 258.7 million. One in four people in Indonesia is a teenager (BKKBN, 2017). The number of teenagers in East Java in 2017 recorded 6, 1 million people (15.64\%) of the population of East Java. Based on gender composition, the number of male adolescents is higher than the number of adolescent girls (51 boys: 49 girls) (BPS, 2017).

According to Erikson, adolescence is a time to reach the level of ego identity and personal identity. Adolescents try to form and show self-identity and characteristics that are unique to themselves. Physical changes are of great concern to adolescents because body image is a mandatory standard that others will see (Wong, 2008). A negative body image creates anxiety for the individual. The negative body image that occurs makes the individual susceptible to body dysmorphic disorders (Dolezal, 2015). This disorder causes the individual to feel that something is lacking in him/ her which the individual looks normal (Denich \& Ifdil, 2015).

Many types of interventions can be done directly to overcome a negative body image to a positive one. One of them is direct intervention through yoga which is very important for the development and maintenance of a positive body image (Jessie E et al., 2011). Intervention can be done indirectly through applications that involve interactive discussions among group members who can overcome eating disorders due to eating patterns that do not match the body's needs to get an ideal body shape (Diao et al., 2020). The purpose of this systematic review is to analyze the effect of psychological interventions on increasing body image in adolescents.

\section{METHODS}

The literature search strategy used five electronic databases, namely Scopus, MEDLINE, CINAHL, Science Direct, and ProQuest. The search was carried out from April-June 2020 to identify relevant research. Keywords in this Systematic review are adjusted to the Medical Subject Heading (MeSH). The keywords used are "Psychological effect" OR " Mental health effect " OR " Mental disorder " OR " Mental problem ", "Intervention" OR " Treatment" OR "Healing " OR "Therapy", "Body image" OR "Body dissatisfaction "OR" Body Representation " and " Adolescents "OR " Teenager " OR " Young adults ". Search is limited to journal articles and in English.

Search is limited to journal articles and in English. Feasibility of the article using PICOS framework. Inclusion criteria: 1) Population, adolescents with psychological 


\section{STRADA Jurnal Ilmiah Kesehatan}

DOI: $10.30994 /$ sjik.v9i2.385

ISSN: 2252-3847 (print); 2614-350X (online)

Vol.9 No.2 November 2020 Page.772-789

problems on body image: healthy, risks and those that have occurred; 2) Intervention, psychological intervention given to respondents, both direct and indirect interventions; 3 ) Comparations, the comparison group used is another intervention or the group that was observed without intervention; 4) Outcome, a study that explains interventions that affect the psychological impact felt by adolescents related to body image; 5) Study design and publication type, randomized controlled trials (RCTs) and quasiexperimental; 6) Publication years, research years 2015 to 2020; 7) Language, English.

Study selection starts from checking for duplicate articles. Then the title, abstract, fulltext, and methodology were screened and assessed for study eligibility. Data tabulation and narrative analysis of the study findings are presented. In assessing the quality of articles, researchers used The Center for review and Dissemination and the Joanna Briggs Institute Guideline. The risk of bias is seen from the research method of each study, which consists of Theory, Design, Samples, Variables, Instruments, and Analysis, and the Prisma checklist as a guide in making this review.

The method of analysis used in this Systematic review is a descriptive method based on the themes that have been determined in the Systematic review. The study uses a descriptive analysis of the research results described in the literature. Relevant data reviewed by review questions, including author, country, year, background, theoretical framework, research objectives, study design, sample size, sampling method, participant descriptions, reliability, and validity, measurement instruments, analysis, and statistical techniques, and analysis of results. The narrative approach collects evidence on the effectiveness of interventions and develops a coherent textual narrative of the similarities and differences between studies, used to synthesize data in this systematic review.

\section{RESULTS}

The results obtained were 17 studies were Randomized Control Trials and 8 studies used Quasy Experimental. Based on these results, after the critical appraisal was carried out using the JBI critical appraisal tools, a quality score was given. Studies using the Randomized Control Trials design were assigned a total score ranging from nine to thirteen points out of a total of thirteen points on the checklist. Pre-Experimental studies on this Systematic review assigned seven to nine points out of a total of nine points on the checklist. The process of extracting and filtering articles is summarized in Figure 1.

Figure 1. Flow diagram and article selection
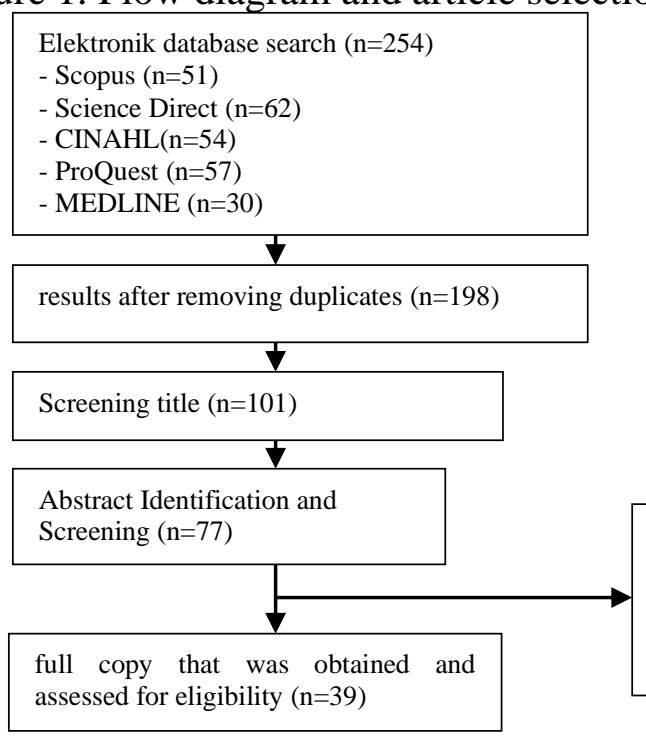

Exclusion $(\mathrm{n}=225)$

- Not RCT or Quasi Experiment

- Not a psychological intervention

- Average age beyond adolescence

- Articles are not in English 


\section{STRADA Jurnal Ilmiah Kesehatan}

DOI: $10.30994 /$ sjik.v9i2.385

ISSN: 2252-3847 (print); 2614-350X (online)

Vol.9 No.2 November 2020 Page.772-789

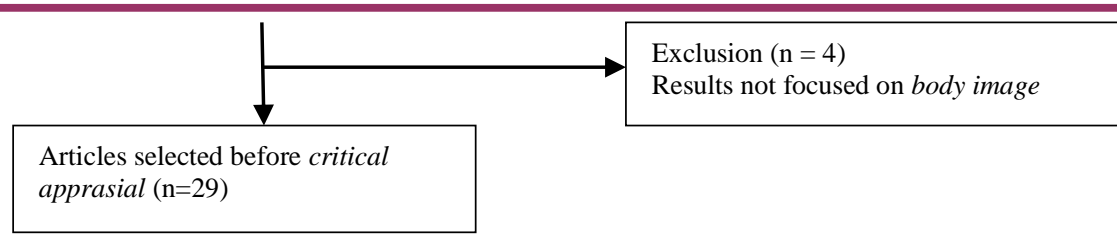

The studies included in this Systematic review article consisted of 7 studies conducted in Australia at universities and research institutes; 3 studies each in the United States, England, and Iran; and 2 studies each in Norway and China. Research and studies were also carried out in Swedish, Italy, Malaysia, Pakistan, and Canada. Respondents in the study had an average age of 11-20 years and were multi-regional. Respondents in this study were adolescents in several countries. Gender characteristics of adolescents in this systematic review are more dominated by women and most of the education levels are at the secondary school level.

Based on the results of the study, it can be grouped according to the specified theme, namely psychological intervention on adolescent body image. The presentation of the results will be determined based on two major themes, a namely psychological intervention which is carried out directly / face to face ( offline ) and indirectly / via the internet ( online ). Based on the results of the study, it was found that the psychological interventions provided were mostly online as many as 13 studies, and the rest were through direct intervention methods. The results of this study can be seen in Table 1 .

Table 1. Characteristics of research articles

\begin{tabular}{|c|c|c|c|c|c|}
\hline $\begin{array}{l}\mathbf{N} \\
\mathbf{0}\end{array}$ & $\begin{array}{l}\text { Author } \\
\text { and Year }\end{array}$ & $\begin{array}{l}\text { Design } \\
\text { Research }\end{array}$ & $\begin{array}{l}\text { Participant } \\
\text { S }\end{array}$ & Intsrumen/ Intervention & Results \\
\hline 1 & $\begin{array}{l}\text { (Seekis et } \\
\text { al., 2020) }\end{array}$ & $\begin{array}{l}\text { Randomiz } \\
\text { ed } \\
\text { Controlled } \\
\text { Trial }\end{array}$ & $\begin{array}{l}76 \text { girls with } \\
\text { average age } \\
18.31 \text { years i } \\
\text { n Australia }\end{array}$ & $\begin{array}{l}\text { The Mindful Self- } \\
\text { Compassion workshop } \\
\text { is complemented by group } \\
\text { discussions on Facebook . } \\
\text { The measuring instruments } \\
\text { used are: 1) Body } \\
\text { Dissatisfaction Subscale } \\
\text { from the Eating Disorders } \\
\text { Inventory -3 (EDI-3); 2) } \\
\text { Drive for Thinness Subscale } \\
\text { from the Eating Disorders } \\
\text { Inventory -3 (EDI ); 3) } \\
\text { Social Appearance Anxiety } \\
\text { Scale (SAAS ); 4) Upward } \\
\text { Physical Appearance } \\
\text { Comparison } \\
\text { Scale (UPACS ); 5) Body } \\
\text { Appreciation Scale- 2 } \\
\text { (BAS-2 ); and 6) Self- } \\
\text { Compassion Scale-Short- } \\
\text { Form (SCS-SF ) }\end{array}$ & $\begin{array}{l}\text { Participants } \\
\text { in group interve } \\
\text { ntion using } \\
\text { the Mindful } \\
\text { Self- } \\
\text { Compassion wh } \\
\text { en experiencing } \\
\text { difficulty in app } \\
\text { earance and } \\
\text { posted on the } \\
\text { experience of } \\
\text { their group Face } \\
\text { book personally } \\
\text { three times per } \\
\text { week for two } \\
\text { weeks. The } \\
\text { findings showed } \\
\text { that the } \\
\text { intervention } \\
\text { group } \\
\text { experienced } \\
\text { a decrease in } \\
\text { anxiety about ap } \\
\text { pearance, body }\end{array}$ \\
\hline
\end{tabular}




\section{STRADA Jurnal Ilmiah Kesehatan}

DOI: $10.30994 /$ sjik.v9i2.385

ISSN: 2252-3847 (print); 2614-350X (online)

Vol.9 No.2 November 2020 Page.772-789

\begin{tabular}{|c|c|c|c|c|c|}
\hline $\begin{array}{l}\mathbf{N} \\
\mathbf{o}\end{array}$ & $\begin{array}{l}\text { Author } \\
\text { and Year }\end{array}$ & $\begin{array}{l}\text { Design } \\
\text { Research }\end{array}$ & $\begin{array}{l}\text { Participant } \\
\text { S }\end{array}$ & Intsrumen/ Intervention & Results \\
\hline & & & & & $\begin{array}{l}\text { dissatisfaction, } \\
\text { and the urge to } \\
\text { be thin, and a } \\
\text { higher body } \\
\text { appreciation and } \\
\text { self-indulgence } \\
\text { on the posttest } \\
\text { and follow-up } \\
\text { one month }\end{array}$ \\
\hline 2 & $\begin{array}{l}\text { (Fogelkvi } \\
\text { st et al., } \\
2020 \text { ) }\end{array}$ & $\begin{array}{l}\text { Randomiz } \\
\text { ed } \\
\text { Controlled } \\
\text { Trial }\end{array}$ & $\begin{array}{l}99 \\
\text { participants } \\
\text { of } \\
\text { outpatients } \\
\text { at clinics in } \\
\text { Sweden } \\
\text { from 2010- } \\
2014\end{array}$ & $\begin{array}{l}\text { Participants were given } 12 \\
\text { sessions consisting of } 2 \\
\text { groups: } \\
\text { ACT: Acceptance and } \\
\text { Commitment Therapy } \\
\text { TAU: Treatment as Usual } \\
\text { The results of the } \\
\text { intervention will be } \\
\text { measured through: 1) Self- } \\
\text { report instruments; 2) } \\
\text { Eating Disorder } \\
\text { Examination } \\
\text { Questionnaire (EDE-Q); 3) } \\
\text { Body Shape } \\
\text { Questionnaire (BSQ); 4) } \\
\text { Body Checking } \\
\text { Questionnaire } \\
\text { (BCQ); 5) Self-Concept } \\
\text { Questionnaire (SCQ); and } \\
\text { 6) Mindful Attention } \\
\text { Awareness Scale (MAAS) }\end{array}$ & $\begin{array}{l}\text { This study supp } \\
\text { ots ACT as a } \\
\text { valid treatment } \\
\text { intervention for } \\
\text { patients with } \\
\text { residual } \\
\text { ED ( Eating } \\
\text { Disorder ) patho } \\
\text { logy and body } \\
\text { image } \\
\text { problems. This } \\
\text { suggests that } \\
\text { ACT is superior } \\
\text { in reducing } \\
\text { body image } \\
\text { problems among } \\
\text { patients with } \\
\text { different ED }\end{array}$ \\
\hline 3 & $\begin{array}{l}\text { (Halliwel } \\
\text { 1 et al., } \\
\text { 2019) }\end{array}$ & $\begin{array}{l}\text { Randomiz } \\
\text { ed } \\
\text { experimen } \\
\text { tal }\end{array}$ & $\begin{array}{l}44 \\
\text { women with } \\
\text { a mean age } \\
\text { of } 20.21 \\
\text { years } \\
\text { with } 61 \% \\
\text { had tried } \\
\text { yoga in the } \\
\text { past, but } \\
\text { only four } \\
\text { participants } \\
\text { currently } \\
\text { participated } \\
\text { in yoga } \\
\text { (three in the }\end{array}$ & $\begin{array}{l}\text { A brief yoga-based body } \\
\text { image intervention that } \\
\text { incorporates } \\
\text { specially designed themes } \\
\text { to focus on positive body } \\
\text { image whose results will be } \\
\text { measured using: } 1 \text { ) The } \\
\text { Body Appreciation Scale- } \\
2 \text { to assess positive body } \\
\text { image; 2) The Positive } \\
\text { Connection with the Body } \\
\text { Subscale of the Experience } \\
\text { of Embodiment Scale; } 3 \text { ) } \\
\text { Body Areas Satisfaction } \\
\text { Subscale (BASS) of the }\end{array}$ & $\begin{array}{l}\text { the intervention } \\
\text { group } \\
\text { reported signific } \\
\text { ant } \\
\text { improvements in } \\
\text { body } \\
\text { appreciation, } \\
\text { bodily } \\
\text { connectedness, } \\
\text { body } \\
\text { satisfaction, and } \\
\text { positive mood at } \\
\text { posttest and at } \\
\text { 4-week follow- } \\
\text { up. }\end{array}$ \\
\hline
\end{tabular}




\section{STRADA Jurnal Ilmiah Kesehatan}

DOI: $10.30994 /$ sjik.v9i2.385

ISSN: 2252-3847 (print); 2614-350X (online)

Vol.9 No.2 November 2020 Page.772-789

\begin{tabular}{|c|c|c|c|c|c|}
\hline $\begin{array}{l}\mathbf{N} \\
\mathbf{0}\end{array}$ & $\begin{array}{l}\text { Author } \\
\text { and Year }\end{array}$ & $\begin{array}{l}\text { Design } \\
\text { Research }\end{array}$ & $\begin{array}{l}\text { Participant } \\
\text { S }\end{array}$ & Intsrumen/ Intervention & Results \\
\hline & & & $\begin{array}{l}\text { control } \\
\text { condition } \\
\text { and one in } \\
\text { the } \\
\text { experimenta } \\
1 \text { condition) }\end{array}$ & $\begin{array}{l}\text { Multidimensional Body- } \\
\text { Self Relations } \\
\text { Questionnaire; 4) The Body } \\
\text { Surveillance Subscale of the } \\
\text { Objectified Body } \\
\text { Consciousness Scale }\end{array}$ & \\
\hline 4 & $\begin{array}{l}\text { (Seekis et } \\
\text { al., 2017) }\end{array}$ & $\begin{array}{l}\text { Randomiz } \\
\text { ed } \\
\text { Controlled } \\
\text { Trial }\end{array}$ & $\begin{array}{l}96 \text { first-year } \\
\text { female } \\
\text { psychology } \\
\text { students at } \\
\text { Australian } \\
\text { universities } \\
\text { with a mean } \\
\text { age of } 19.45 \\
\text { years }\end{array}$ & $\begin{array}{l}\text { Participants were randomly } \\
\text { divided into three writing } \\
\text { treatment groups: self- } \\
\text { compassion, self-esteem, } \\
\text { and control. } \\
\text { The results of the } \\
\text { intervention will be } \\
\text { measured by: 1) State Body } \\
\text { Appreciation Scale-2 } \\
\text { Body Image States Scale } \\
\text { (BISS ); 2) The Physical } \\
\text { Appearance State and Trait } \\
\text { Anxiety Scale - } \\
\text { state version (PASTAS ) }\end{array}$ & $\begin{array}{l}\text { Self-compassion } \\
\text { writing intervent } \\
\text { ion has a } \\
\text { considerable infl } \\
\text { uence as a } \\
\text { means } \\
\text { helps people in e } \\
\text { liminating negat } \\
\text { ive body image }\end{array}$ \\
\hline 5 & $\begin{array}{l}\text { (Wilksch } \\
\text { et al., } \\
\text { 2018) }\end{array}$ & $\begin{array}{l}\text { Randomiz } \\
\text { ed } \\
\text { Controlled } \\
\text { Trial }\end{array}$ & $\begin{array}{l}575 \text { women } \\
\text { aged } 18-25 \\
\text { years from } \\
\text { Australia } \\
\text { and New } \\
\text { Zealand }\end{array}$ & $\begin{array}{l}\text { Participants were randomly } \\
\text { divided into three groups : } \\
\text { 1) Smart-Targeted Media } \\
\text { (MS-T); 2) Student Bodies } \\
\text { (SB); 3) Control. } \\
\text { The results of the } \\
\text { intervention will be } \\
\text { measured using the Eating } \\
\text { Disorder Examination } \\
\text { Questionnaire (EDE-Q ). }\end{array}$ & $\begin{array}{l}\text { MS-T has the } \\
\text { potential to } \\
\text { achieve risk } \\
\text { reduction in wo } \\
\text { men with ED } \\
\text { ( Disordered E a } \\
\text { ting ) affecting } \\
\text { body image at } \\
\text { low } \\
\text { implementation } \\
\text { costs. }\end{array}$ \\
\hline 6 & $\begin{array}{l}\text { (Maasou } \\
\text { meh et } \\
\text { al., 2020) }\end{array}$ & $\begin{array}{l}\text { quasi- } \\
\text { experimen } \\
\text { tal }\end{array}$ & $\begin{array}{l}60 \text { female } \\
\text { students of } \\
\text { grade } 6 \mathrm{SD} \\
\text { in Yazd } \\
\text { (Iran) aged } \\
12.16 \text { years }\end{array}$ & $\begin{array}{l}\text { The intervention group } \\
\text { was educated at school for } 8 \\
\text { sessions x } 45 \text { minutes per } \\
\text { session based on HBM, } \\
\text { while the control group was } \\
\text { educated using the } \\
\text { traditional lecture } \\
\text { method . Two groups were } \\
\text { assessed with } \\
\text { the Multidimensional Body } \\
\text { - Self Relations } \\
\text { Questionnaire (MBSRQ) at } \\
\text { pretest and posttest. }\end{array}$ & $\begin{array}{l}\text { The effectivenes } \\
\text { s and efficiency } \\
\text { of puberty based } \\
\text { health teaching } \\
\text { HBM ( Health } \\
\text { Belief } \\
\text { Model ) on } \\
\text { improving body } \\
\text { image that is felt } \\
\text { in adolescent } \\
\text { women }\end{array}$ \\
\hline 7 & (Christie & randomise & 174 children & The intervention group was & Intervention $\mathrm{HE}$ \\
\hline
\end{tabular}




\section{STRADA Jurnal Ilmiah Kesehatan}

DOI: $10.30994 /$ sjik.v9i2.385

ISSN: 2252-3847 (print); 2614-350X (online)

Vol.9 No.2 November 2020 Page.772-789

\begin{tabular}{|c|c|c|c|c|c|}
\hline $\begin{array}{l}\mathbf{N} \\
\mathbf{0}\end{array}$ & $\begin{array}{l}\text { Author } \\
\text { and Year }\end{array}$ & $\begin{array}{l}\text { Design } \\
\text { Research }\end{array}$ & $\begin{array}{l}\text { Participant } \\
\text { s }\end{array}$ & Intsrumen/ Intervention & Results \\
\hline & $\begin{array}{l}\text { et al., } \\
\text { 2017) }\end{array}$ & $\begin{array}{l}\text { d } \\
\text { controlled } \\
\text { trial }\end{array}$ & $\begin{array}{l}\text { aged } 13-17 \\
\text { in the UK }\end{array}$ & $\begin{array}{l}\text { given HELP } \\
\text { intervention. HELP is a } \\
\text { family-based weight } \\
\text { management consisting of } \\
12 \text { sessions } \\
\text { program for teenagers for } 5 \\
\text { days to change behavior by } \\
\text { approaching and motivating }\end{array}$ & $\begin{array}{l}\text { LP no more } \\
\text { effective } \\
\text { than the one } \\
\text { educational } \\
\text { sessions individ } \\
\text { ual to reduce } \\
\text { BMI ( Body } \\
\text { Mass Index) in } \\
\text { an obese sample } \\
\text { of adolescents in } \\
\text { communities w } \\
\text { here a suitable } \\
\text { BMI will } \\
\text { maintain a } \\
\text { positive body } \\
\text { image. }\end{array}$ \\
\hline 8 & $\begin{array}{l}\text { (Diao et } \\
\text { al., 2020) }\end{array}$ & $\begin{array}{l}\text { Randomiz } \\
\text { ed } \\
\text { Controlled } \\
\text { Trial } \\
\text { (RCT) }\end{array}$ & $\begin{array}{l}948 \text { particip } \\
\text { ants ( } 479 \\
\text { boys and } \\
469 \text { girls, } \\
642 \text { of } \\
\text { whom were } \\
\text { elementary } \\
\text { school } \\
\text { students and } \\
306 \text { high } \\
\text { school } \\
\text { students) div } \\
\text { ided into } \\
\text { the } \\
\text { intervention } \\
\text { group (n } \\
=518) \text { and } \\
\text { the control } \\
\text { group (n = } \\
430)\end{array}$ & $\begin{array}{l}\text { The intervention group } \\
\text { received } 1 \text { year of obesity- } \\
\text { related health education, } \\
\text { physical exercise, and diet } \\
\text { control. Their baseline body } \\
\text { mass index (BMI) was } \\
\text { calculated, their quality } \\
\text { of life and baseline } \\
\text { information were assessed } \\
\text { before and after the } \\
\text { intervention period using } \\
\text { the self- } \\
\text { designed Adolescent } \\
\text { Quality of Life } \\
\text { Scale and baseline } \\
\text { information questionnaire }\end{array}$ & $\begin{array}{l}\text { Family- } \\
\text { individual- } \\
\text { school-based } \\
\text { interventions } \\
\text { combining } \\
\text { obesity-related } \\
\text { health } \\
\text { education, physi } \\
\text { cal exercise, } \\
\text { and diet control } \\
\text { can improve } \\
\text { psychological } \\
\text { quality, puberty, } \\
\text { and total } \\
\text { QoL ( Quality of } \\
\text { Life ). }\end{array}$ \\
\hline 9 & $\begin{array}{l}\text { (Lee et } \\
\text { al., 2017) }\end{array}$ & $\begin{array}{l}\text { Randomiz } \\
\text { ed } \\
\text { Controlled } \\
\text { Trial } \\
\text { (RCT) }\end{array}$ & $\begin{array}{l}115 \text { students } \\
\text { (interventio } \\
\text { n group }= \\
63 \text { ) and } \\
\text { control } \\
\text { group }=52 \text { ) } \\
\text { with a mean } \\
\text { age of } 11.4\end{array}$ & $\begin{array}{l}\text { School based } \\
\text { weight program } \\
\text { (SBWMP) involve parents } \\
\text { through mHealth tools are } \\
\text { designed to reduce weight, } \\
\text { improve their knowledge } \\
\text { and adopt a healthy lifestyle } \\
\text { that target children and }\end{array}$ & $\begin{array}{l}\text { There is a } \\
\text { positive and } \\
\text { direct impact of } \\
\text { the SBWMP on } \\
\text { students' health } \\
\text { knowledge and } \\
\text { a psychological } \\
\text { impact on the }\end{array}$ \\
\hline
\end{tabular}




\section{STRADA Jurnal Ilmiah Kesehatan}

DOI: $10.30994 /$ sjik.v9i2.385

ISSN: 2252-3847 (print); 2614-350X (online)

Vol.9 No.2 November 2020 Page.772-789

\begin{tabular}{|c|c|c|c|c|c|}
\hline $\begin{array}{l}\mathbf{N} \\
\mathbf{0}\end{array}$ & $\begin{array}{l}\text { Author } \\
\text { and Year }\end{array}$ & $\begin{array}{l}\text { Design } \\
\text { Research }\end{array}$ & $\begin{array}{l}\text { Participant } \\
\text { s }\end{array}$ & Intsrumen/ Intervention & Results \\
\hline & & & $\begin{array}{l}\text { years in four } \\
\text { schools }\end{array}$ & $\begin{array}{l}\text { adolescents with } \\
\text { obesity with Mild } \\
\text { Intellectual D isabilities (MI } \\
\text { Ds). }\end{array}$ & $\begin{array}{l}\text { intervention } \\
\text { group. SBWMP } \\
\text { involving } \\
\text { parents } \\
\text { through mHealt } \\
\mathrm{h} \text { is a viable and } \\
\text { acceptable } \\
\text { program } \\
\text { for students with } \\
\text { MID } \\
\text { and their parents }\end{array}$ \\
\hline 10 & $\begin{array}{l}\text { (Ishak et } \\
\text { al., 2016) }\end{array}$ & $\begin{array}{l}\text { Quasy } \\
\text { eksperime } \\
\text { n }\end{array}$ & $\begin{array}{l}\text { high school } \\
\text { teens first ag } \\
\text { ed 13-14 } \\
\text { years }\end{array}$ & $\begin{array}{l}\text { Eat Right, Be Positive } \\
\text { About Your Body and Live } \\
\text { Actively (EPaL) was } \\
\text { promoted were healthy } \\
\text { eating, positive body image } \\
\text { and an active } \\
\text { lifestyle. Interventions will } \\
\text { be assessed by the } \\
\text { perception of one's body } \\
\text { weight } \\
\text { status, Body } \\
\text { Dissatisfaction subscale and } \\
\text { Body Importance subscale } \\
\text { extracted from the Body } \\
\text { Image and Body Change } \\
\text { Inventory, and the Body } \\
\text { Image Scale. }\end{array}$ & $\begin{array}{l}\text { EPAL will } \\
\text { contribute to } \\
\text { preventing } \\
\text { overweight and } \\
\text { disorders } \\
\text { eating with a } \\
\text { positive effect } \\
\text { on weight status, } \\
\text { healthy lifestyle } \\
\text { behavior, and } \\
\text { quality of life } \\
\text { related to } \\
\text { health, thereby } \\
\text { creating a } \\
\text { positive body } \\
\text { image. }\end{array}$ \\
\hline 11 & $\begin{array}{l}\text { (Goldfiel } \\
\text { d et al., } \\
2015)\end{array}$ & $\begin{array}{l}\text { Randomiz } \\
\text { ed } \\
\text { Controlled } \\
\text { Trial } \\
\text { (RCT) }\end{array}$ & $\begin{array}{l}304 \\
\text { adolescents } \\
\text { (91 boys, } \\
213 \text { girls) } \\
\text { with } 93 \% \\
\text { being obese } \\
\text { over the age } \\
\text { of } 14-18 \\
\text { years }\end{array}$ & $\begin{array}{l}\text { All adolescents receive } \\
\text { counseling and participate } \\
\text { in sports } 4 \text { times a week for } \\
22 \text { weeks which are divided } \\
\text { into } 4 \text { groups where the } \\
\text { results will be measured } \\
\text { using: 1) Brunel Mood } \\
\text { Scale for mood; 2) Multiple } \\
\text { Body Self-Relations } \\
\text { Questionnaire for body } \\
\text { image; 3) Harter Physical } \\
\text { Self-Perceptions; } 4 \text { ) } \\
\text { Questionnaire for physical } \\
\text { self-perception and self } \\
\text { esteem }\end{array}$ & $\begin{array}{l}\text { All groups } \\
\text { showed } \\
\text { improved body } \\
\text { image and } \\
\text { improved } \\
\text { physical self- } \\
\text { perception }\end{array}$ \\
\hline 12 & (Rodgers & Quasy & 274 & BodiMojo which is based at & There are \\
\hline
\end{tabular}




\section{STRADA Jurnal Ilmiah Kesehatan}

DOI: $10.30994 /$ sjik.v9i2.385

ISSN: 2252-3847 (print); 2614-350X (online)

Vol.9 No.2 November 2020 Page.772-789

\begin{tabular}{|c|c|c|c|c|c|}
\hline $\begin{array}{l}\mathbf{N} \\
\mathbf{0}\end{array}$ & $\begin{array}{l}\text { Author } \\
\text { and Year }\end{array}$ & $\begin{array}{l}\text { Design } \\
\text { Research }\end{array}$ & $\begin{array}{l}\text { Participant } \\
\text { s }\end{array}$ & Intsrumen/ Intervention & Results \\
\hline & $\begin{array}{l}\text { et al., } \\
\text { 2018) }\end{array}$ & $\begin{array}{l}\text { experimen } \\
\mathrm{t}\end{array}$ & $\begin{array}{l}\text { adolescents } \\
\text { with an } \\
\text { average age } \\
\text { of } 18.36 \text { and } \\
74 \% \text { were } \\
\text { girls }\end{array}$ & $\begin{array}{l}\text { the mercy of themselves to } \\
\text { promote positive body } \\
\text { image for six } \\
\text { weeks. Interventions will be } \\
\text { assessed with The Self- } \\
\text { Compassion } \\
\text { Scale (SCS ), The Physical } \\
\text { Appearance Comparison } \\
\text { Scale, The Body Image- } \\
\text { Acceptance and Action } \\
\text { Questionnaire (BIAAQ), } \\
\text { and The Positive and } \\
\text { Negative Affect Schedule } \\
\text { 10-Children (HOT-C10) }\end{array}$ & $\begin{array}{l}\text { evidence early } \\
\text { use of } \\
\text { Applications } \\
\text { BodiMojo } \\
\text { associated with } \\
\text { an increase in } \\
\text { the price of self } \\
\text { and self- } \\
\text { compassion }\end{array}$ \\
\hline 13 & $\begin{array}{l}\text { (Tirlea et } \\
\text { al., 2016) }\end{array}$ & $\begin{array}{l}\text { randomize } \\
\mathrm{d} \\
\text { controlled } \\
\text { trial }\end{array}$ & $\begin{array}{l}122 \text { primary } \\
\text { and } \\
\text { secondary } \\
\text { school girls } \\
\text { aged } 10 \text { - } 16 \\
\text { years }\end{array}$ & $\begin{array}{l}\text { Girls on the Go! is a 10- } \\
\text { week program designed to } \\
\text { improve self-esteem, body } \\
\text { image, and self- } \\
\text { confidence using an } \\
\text { empowerment model that } \\
\text { involves interactive and } \\
\text { experiential learning } \\
\text { approaches . Measurements } \\
\text { were made using } \\
\text { the Rosenberg Self-Esteem } \\
\text { Scale, clinical interview } \\
\text { assessment, health self- } \\
\text { efficacy } \\
\text { (including mental health } \\
\text { and physical health self- } \\
\text { efficacy scales ), body } \\
\text { esteem scale, and the } \\
\text { Dutch Eating Behavior } \\
\text { Questionnaire for Children. }\end{array}$ & $\begin{array}{l}\text { Intervention } \\
\text { cause a } \\
\text { significant } \\
\text { increase in self- } \\
\text { esteem and self- } \\
\text { efficacy (subscal } \\
\text { e self-efficacy } \\
\text { physical and } \\
\text { mental } \\
\text { health) for } \\
\text { primary school- } \\
\text { age participants } \\
\text { and reduce } \\
\text { dietary } \\
\text { behavior in } \\
\text { participants high } \\
\text { school. }\end{array}$ \\
\hline 14 & $\begin{array}{l}\text { (Stapleto } \\
\text { n et al., } \\
2016)\end{array}$ & $\begin{array}{l}\text { randomize } \\
\text { d } \\
\text { controlled } \\
\text { trial }\end{array}$ & $\begin{array}{l}22 \\
\text { participants } \\
\text { aged 14-15 } \\
\text { years }\end{array}$ & $\begin{array}{l}\text { The results of the } \\
\text { intervention will be } \\
\text { assessed with The Youth } \\
\text { Adolescent Food Frequency } \\
\text { Questionnaire - Short } \\
\text { Version (YAQ ), Rosenberg } \\
\text { Self-Esteem Scale (RSEQ), } \\
\text { Depression Anxiety Stress } \\
\text { Scal e - } 21 \text { (DASS-21), } \\
\text { and Self-Compassion } \\
\text { Scale (SCS ). }\end{array}$ & $\begin{array}{l}\text { EFT as an } \\
\text { effective } \\
\text { treatment } \\
\text { strategy to } \\
\text { promote healthy } \\
\text { eating behavior } \\
\text { and improve } \\
\text { weight } \\
\text { psychopatholog } \\
\text { y }\end{array}$ \\
\hline
\end{tabular}




\section{STRADA Jurnal Ilmiah Kesehatan}

DOI: $10.30994 /$ sjik.v9i2.385

ISSN: 2252-3847 (print); 2614-350X (online)

Vol.9 No.2 November 2020 Page.772-789

\begin{tabular}{|c|c|c|c|c|c|}
\hline $\begin{array}{l}\mathbf{N} \\
\mathbf{0}\end{array}$ & $\begin{array}{l}\text { Author } \\
\text { and Year }\end{array}$ & $\begin{array}{l}\text { Design } \\
\text { Research }\end{array}$ & $\begin{array}{l}\text { Participant } \\
\text { S }\end{array}$ & Intsrumen/ Intervention & Results \\
\hline 15 & $\begin{array}{l}\text { (Krebs et } \\
\text { al., 2017) }\end{array}$ & $\begin{array}{l}\text { randomize } \\
\mathrm{d} \\
\text { controlled } \\
\text { trial }\end{array}$ & $\begin{array}{l}26 \\
\text { adolescents } \\
\text { with a mean } \\
\text { age of } 16.2 \\
\text { years }\end{array}$ & $\begin{array}{l}\text { The intervention group with } \\
\text { a primary diagnosis of BDD } \\
\text { ( Body D ysmorphic D isor } \\
\text { der ) received CBT } \\
\text { intervention for } 14 \text { sessions } \\
\text { and was followed up for } 12 \\
\text { months (2, 6, and } 12 \\
\text { months) }\end{array}$ & $\begin{array}{l}\text { The intervention } \\
\text { group was able } \\
\text { to maintain a } \\
\text { positive and } \\
\text { consistent body } \\
\text { image }\end{array}$ \\
\hline 16 & $\begin{array}{l}\text { (Dunstan } \\
\text { et al., } \\
\text { 2016) }\end{array}$ & $\begin{array}{l}\text { randomize } \\
\mathrm{d} \\
\text { controlled } \\
\text { trial }\end{array}$ & $\begin{array}{l}200 \\
\text { girls with a } \\
\text { mean age of } \\
12.66 \text { years }\end{array}$ & $\begin{array}{l}\text { Six intervention } \\
\text { session Happy Being Me } \\
\text { Coedukasi designed to } \\
\text { reduce risk factors and body } \\
\text { dissatisfaction in girls. The } \\
\text { results of the intervention } \\
\text { will be assessed using } \\
\text { the Eating Disorder } \\
\text { Inventory - Body } \\
\text { Dissatisfaction } \\
\text { Subscale (EDI- } \\
\text { BD ), Physical Appearance } \\
\text { Comparison } \\
\text { Scale, Rosenberg Self- } \\
\text { Esteem Scale (RSES ), } \\
\text { and Appearance } \\
\text { Conversations Scale }\end{array}$ & $\begin{array}{l}\text { HBM Co-Ed } \\
\text { was effective in } \\
\text { reducing } \\
\text { psychological } \\
\text { risk factors for } \\
\text { body } \\
\text { dissatisfaction } \\
\text { during the six- } \\
\text { month follow-up } \\
\text { period and } \\
\text { postintervention } \\
\text { of body } \\
\text { dissatisfaction in } \\
\text { girls. }\end{array}$ \\
\hline 17 & $\begin{array}{l}\text { (Christine } \\
\text { et al., } \\
2018)\end{array}$ & $\begin{array}{l}\text { randomize } \\
\text { d } \\
\text { controlled } \\
\text { trial }\end{array}$ & $\begin{array}{l}2481 \text { grade } \\
12 \text { students } \\
\text { from } 30 \\
\text { schools }\end{array}$ & $\begin{array}{l}\text { The intervention consists of } \\
\text { three workshops, each } 90 \\
\text { minutes to the main theme, } \\
\text { namely body image, media } \\
\text { literacy, and } \\
\text { lifestyle. Interventions are } \\
\text { interactive and are led by } \\
\text { trained scientists }\end{array}$ & $\begin{array}{l}\text { Healthy Body } \\
\text { image } \\
\text { Hopefully it can } \\
\text { prove its } \\
\text { efficacy }\end{array}$ \\
\hline 18 & $\begin{array}{l}\text { (Toole \& } \\
\text { Craighea } \\
\text { d, 2016) }\end{array}$ & $\begin{array}{l}\text { Nonrando } \\
\text { mised } \\
\text { controlled } \\
\text { study }\end{array}$ & $\begin{array}{l}87 \text { women } \\
\text { had a mean } \\
\text { age of } 18.85 \\
\text { years with } \\
\text { body } \\
\text { dissatisfacti } \\
\text { on }\end{array}$ & $\begin{array}{l}\text { Internet-based self- } \\
\text { compassion training for } 1 \\
\text { week to improve aspects } \\
\text { of self-compassion and } \\
\text { BID ( Body image } \\
\text { distress ). Interventions will } \\
\text { be assessed by BMI, The } \\
\text { Self-Compassion } \\
\text { Scale, Body Appreciation } \\
\text { Scale, Rosenberg Self- } \\
\text { Esteem Scale, Body }\end{array}$ & $\begin{array}{l}\text { There is } \\
\text { significant in the } \\
\text { appreciation of } \\
\text { the shape of the } \\
\text { body and self- } \\
\text { confidence for } \\
\text { the intervention } \\
\text { group compared } \\
\text { with the control } \\
\text { group. }\end{array}$ \\
\hline
\end{tabular}




\section{STRADA Jurnal Ilmiah Kesehatan}

DOI: $10.30994 /$ sjik.v9i2.385

ISSN: 2252-3847 (print); 2614-350X (online)

Vol.9 No.2 November 2020 Page.772-789

\begin{tabular}{|c|c|c|c|c|c|}
\hline $\begin{array}{l}\mathbf{N} \\
\mathbf{o}\end{array}$ & $\begin{array}{l}\text { Author } \\
\text { and Year }\end{array}$ & $\begin{array}{l}\text { Design } \\
\text { Research }\end{array}$ & $\begin{array}{l}\text { Participant } \\
\text { s }\end{array}$ & Intsrumen/ Intervention & Results \\
\hline & & & & $\begin{array}{l}\text { Surveillance subscale of } \\
\text { the Objectified } \\
\text { Body Consciousness Scale, } \\
\text { Contingencies of Self- } \\
\text { Worth Scale-Appearance } \\
\text { Subscale, and Body Shape } \\
\text { Questionnaire }\end{array}$ & \\
\hline 19 & $\begin{array}{l}\text { (Wolfe \& } \\
\text { Patterson, } \\
2017 \text { ) }\end{array}$ & $\begin{array}{l}\text { Nonrando } \\
\text { mised } \\
\text { controlled } \\
\text { study }\end{array}$ & $\begin{array}{l}108 \text { female } \\
\text { students } \\
\text { with an } \\
\text { average age } \\
\text { of } 20.44 \\
\text { years }\end{array}$ & $\begin{array}{l}\text { Intervention } \\
\text { gratitude with BD ( body } \\
\text { dissatisfaction ) together } \\
\text { with a common sequelae of } \\
\text { BD: can not eat } \\
\text { properly, negative mood, } \\
\text { and depressive symptoms. } \\
\text { Interventions will be } \\
\text { assessed using the Body } \\
\text { Shape Questionnaire } \\
\text { (BSQ), Attitudes Test-26 } \\
\text { (EAT) and the Binge Eating } \\
\text { Scale (BINGE), and } \\
\text { the Positive and Negative } \\
\text { Affect Scale (HOT) and the } \\
\text { Center for Epidemiologic } \\
\text { Studies Depression Scale } \\
\text { (CES-D) }\end{array}$ & $\begin{array}{l}\text { The intervention } \\
\text { group in } \\
\text { the grateful cate } \\
\text { gory was } \\
\text { significantly abl } \\
\text { e to increase } \\
\text { body reward } \\
\text { compared to the } \\
\text { control group }\end{array}$ \\
\hline 20 & $\begin{array}{l}\text { (Givehki } \\
\text { et al., } \\
2018 \text { ) }\end{array}$ & $\begin{array}{l}\text { randomize } \\
\text { d clinical } \\
\text { trial }\end{array}$ & $\begin{array}{l}75 \text { people w } \\
\text { ith physical } \\
\text { pain due to } \\
\text { psychologic } \\
\text { al } \\
\text { (emotional } \\
\text { or mental) } \\
\text { disorders }\end{array}$ & $\begin{array}{l}\text { Respondents who were } \\
\text { randomly selected were } \\
\text { divided into } \\
\text { three group members . } \\
\text { Intervention will be } \\
\text { assessed using B ody-Image } \\
\text { Acceptance and Action } \\
\text { Questionnaire (BI- } \\
\text { AAQ), Body awareness } \\
\text { questionnaire (BAQ), } \\
\text { and Psychosomatic } \\
\text { disorders by DSM-5. }\end{array}$ & $\begin{array}{l}\text { ACT ( Acceptan } \\
\text { ce and } \\
\text { Commitment } \\
\text { Therapy ) mana } \\
\text { ged to increase } \\
\text { the flexibility of } \\
\text { the } \\
\text { picture 's body } \\
\text { and } \\
\text { consciousness } \\
\text { of the patient } \\
\text { on the body of } \\
\text { her with } \\
\text { physical pain } \\
\text { due to } \\
\text { psychological } \\
\text { disorders } \\
\text { (emotions or } \\
\text { thoughts) }\end{array}$ \\
\hline 21 & (Sundgot- & randomize & 2,446 grade & The Healthy Body & The HBI \\
\hline
\end{tabular}




\section{STRADA Jurnal Ilmiah Kesehatan}

DOI: $10.30994 /$ sjik.v9i2.385

ISSN: 2252-3847 (print); 2614-350X (online)

Vol.9 No.2 November 2020 Page.772-789

\begin{tabular}{|c|c|c|c|c|c|}
\hline $\begin{array}{l}\mathbf{N} \\
\mathbf{0}\end{array}$ & $\begin{array}{l}\text { Author } \\
\text { and Year }\end{array}$ & $\begin{array}{l}\text { Design } \\
\text { Research }\end{array}$ & $\begin{array}{l}\text { Participant } \\
\text { s }\end{array}$ & Intsrumen/ Intervention & Results \\
\hline & $\begin{array}{l}\text { borgen et } \\
\text { al., 2019) }\end{array}$ & $\begin{array}{l}\mathrm{d} \text { clinical } \\
\text { trial }\end{array}$ & $\begin{array}{l}12 \text { boys and } \\
\text { girls with a } \\
\text { mean age of } \\
16.8 \text { years } \\
\text { from } 30 \\
\text { secondary } \\
\text { schools }\end{array}$ & $\begin{array}{l}\text { Image (HBI) intervention } \\
\text { was conducted for } \\
\text { positive health - } \\
\text { related quality of } \\
\text { life among secondary } \\
\text { school students. } \\
\text { Data collected at baseline, } \\
\text { post intervention, } 3 \text { and } 12 \\
\text { months for follow-up were } \\
\text { analyzed using mixed } \\
\text { regression models. }\end{array}$ & $\begin{array}{l}\text { intervention led } \\
\text { to } \\
\text { direct, favorable } \\
\text { changes in } \\
\text { positive health- } \\
\text { related quality } \\
\text { of life among } \\
\text { the women's } \\
\text { intervention } \\
\text { group versus the } \\
\text { men's } \\
\text { intervention } \\
\text { group that was } \\
\text { maintained at } \\
\text { follow-up. }\end{array}$ \\
\hline 22 & $\begin{array}{l}\text { (Tanofsk } \\
\text { y-kraff et } \\
\text { al., 2017) }\end{array}$ & $\begin{array}{l}\text { randomize } \\
\text { d clinical } \\
\text { trial }\end{array}$ & $\begin{array}{l}113 \\
\text { adolescent } \\
\text { girls aged } \\
12-17 \text { years } \\
\text { who are at } \\
\text { risk of } \\
\text { obesity }\end{array}$ & $\begin{array}{l}\text { Interpersonal Psychotherap } \\
\text { y (IPT) is given to compare } \\
\text { the effectiveness of IPT } \\
\text { with Health Education (HE) } \\
\text { in reducing anxiety about } \\
\text { body shape and body image } \\
\text { of adolescents. }\end{array}$ & $\begin{array}{l}\text { IPT is not } \\
\text { superior to HE } \\
\text { for reducing } \\
\text { anxiety and } \\
\text { improving } \\
\text { adolescent body } \\
\text { image }\end{array}$ \\
\hline 23 & $\begin{array}{l}\text { (Khoshke } \\
\text { rdar \& } \\
\text { Raeisi, } \\
\text { 2020) }\end{array}$ & $\begin{array}{l}\text { Quasy } \\
\text { eksperime } \\
\text { nt }\end{array}$ & $\begin{array}{l}30 \text { girls } \\
\text { aged 16-18 } \\
\text { years }\end{array}$ & $\begin{array}{l}\text { The experimental group } \\
\text { received MBS R } \\
\text { ( Mindfulness-Based Stress } \\
\text { Reduction) intervention } \\
\text { for } 8 \text { sessions x } 90 \text { minutes. } \\
\text { Data were collected using } \\
\text { the Eating Attitude } \\
\text { Test and Body image } \\
\text { Concern Inventory }\end{array}$ & $\begin{array}{l}\text { MBSR can be } \\
\text { effective in } \\
\text { enhancing the } \\
\text { body image of } \\
\text { adolescent girls } \\
\text { with } \\
\text { dysfunctional } \\
\text { eating behavior }\end{array}$ \\
\hline 24 & $\begin{array}{l}\text { (Diedrich } \\
\text { s et al., } \\
2015)\end{array}$ & $\begin{array}{l}\text { randomize } \\
\text { d clinical } \\
\text { trial }\end{array}$ & $\begin{array}{l}1707 \\
\text { adolescents } \\
\text { aged } 11-13 \\
\text { years with } \\
50.83 \% \\
\text { female }\end{array}$ & $\begin{array}{l}\text { The intervention was } \\
\text { carried out using } \\
\text { discussions, small groups, } \\
\text { and video for } 90 \text { minutes } \\
\text { with one provider for about } \\
25-30 \text { students. }\end{array}$ & $\begin{array}{l}\text { The intervention } \\
\text { has short-term } \\
\text { benefits for } \\
\text { body image }\end{array}$ \\
\hline 25 & $\begin{array}{l}\text { (Marengo } \\
\text { et al., } \\
2018)\end{array}$ & $\begin{array}{l}\text { Quasy } \\
\text { eksperime } \\
\text { nt }\end{array}$ & $\begin{array}{l}523 \\
\text { adolescents } \\
\text { with a mean } \\
\text { age of } 14.82 \\
\text { years }\end{array}$ & $\begin{array}{l}\text { Teenagers who } \\
\text { use Facebook every hour of } \\
\text { the day and highly } \\
\text { visual social } \\
\text { media (HVSM) such as } \\
\text { Instagram and Snapchat. } \\
\text { Participants were asked to } \\
\text { complete the BSQ ( Body }\end{array}$ & $\begin{array}{l}\text { Teenagers } \\
\text { reported } \\
\text { pengg unaan } \\
\text { HVSM } \\
\text { that high- } \\
\text { risk increased ri } \\
\text { ght image } \\
\text { problems }\end{array}$ \\
\hline
\end{tabular}




\section{STRADA Jurnal Ilmiah Kesehatan}

DOI: $10.30994 /$ siik.v9i2.385

ISSN: 2252-3847 (print); 2614-350X (online)

Vol.9 No.2 November 2020 Page.772-789

\begin{tabular}{lllll}
\hline $\begin{array}{l}\text { N } \\
\text { o } \\
\text { and Year }\end{array}$ & $\begin{array}{l}\text { Design } \\
\text { Research }\end{array}$ & $\begin{array}{l}\text { Participant } \\
\text { s }\end{array}$ & Intsrumen/ Intervention & Results \\
\hline & & & Shape Questionnaire ) & Tubu h which in \\
& & questionnaire and the Italian & $\begin{array}{l}\text { Turn can cause } \\
\text { self-rated version of the }\end{array}$ & a condition psyc \\
& & Strength and Difficulties & hologically \\
& & & Questionnaires (SDQ) & worse \\
\hline
\end{tabular}

\section{DISCUSSION}

\section{Indirect Psychological Intervention ( Online )}

The results of the review show that there is an effect of an indirect (online) intervention on increasing negative body image to positive and maintaining a positive body image. Interventions involving interactive discussions among group members show promising results for overcoming negative body images. This approach also provides an opportunity for data collection and program evaluation online, including allowing participants to choose a place and time convenient to express what is experienced. This is impacting on self-compassion that serves as a useful framework for intervention line (Rodgers et al., 2018). Self-compassion is providing understanding and kindness to oneself when facing suffering, experiencing failure or making mistakes by not judging one's shortcomings, imperfections, failures, and acknowledging that one's own experiences are part of general human experience (Georgakaki \& Karakasidou, 2017). Interventions targeting body image have been deployed for individual use, for example, meditation, applications, and writing tasks (Seekis et al., 2017).

The results of reviews of several journals explain several types of interventions that can be carried out using internet technology. Some interventions focus on a more profound cognitive component (eg internalization of media and ineffectiveness). It seeks to reduce the risk and prevention of ED (Eating Disorder) with lower implementation costs (Wilksch et al., 2018). There is a direct positive impact of the intervention which indirectly is an increase in quality of life, self-esteem, body shape rating scale, self-efficacy, student health knowledge, and psychological impact on the intervention group (Lee et al., 2017). Online interventions that can be done to improve self-compassion to achieve a positive body image (Rodgers et al., 2018).

The family-based weight management intervention developed focuses on behavior change techniques so that clients can increase a positive body image with family support and monitoring (Christie et al., 2017). Training using online applications and media can reduce several forms of body dissatisfaction including the symptoms of BDD ( Body Dysmorphic Disorder ) in women who are at high risk of developing BID ( Body image Disorder) (Cerea et al., 2020).

Factors that can support the effectiveness of the intervention to increase body image in adolescents are the level of education, age, and gender of the individual. A person's acceptance of new and easy things in terms of self-adjustment as the level of education increases (Notoatmodjo, 2010). This statement where individuals are increasingly receptive to information along with higher education so that there will be more knowledge (Mandias, 2012). This has an impact, where if low education will hinder the development of their behavior towards the acceptance of new information and knowledge.

\section{Indirect Psychological Intervention ( Offline )}




\section{STRADA Jurnal Ilmiah Kesehatan}

DOI: $10.30994 /$ sjik.v9i2.385

ISSN: 2252-3847 (print); 2614-350X (online)

Vol.9 No.2 November 2020 Page.772-789

Indirect / face-to-face ( offline ) psychological intervention has been shown to reduce anxiety in appearance, dissatisfaction with body shape, and higher body appreciation. One intervention asked participants to discuss their daily experiences related to their body image (Seekis et al., 2020). Excessive assessment and control of body shape and weight can lead to eating disorders where interventions made through app intermediaries are very helpful for individuals with eating disorders because their behavior is often aimed at avoiding or controlling inner experiences. Methods used to control thoughts and feelings are used to limit food intake and increase the desire to eat a lot (Chairani, 2018).

One of the direct interventions, ACT asks patients to clarify their values, which aim to increase motivation for behavior change (Fogelkvist et al., 2020). Mindfulness and acceptance of inner experiences help the individual to change behaviors that should be avoided or to control painful mental states (Trindade et al., 2018). Intervention writing tasks were also carried out with no interaction between participants. The results of this intervention show a considerable effect in eliminating negative body image (Seekis et al., 2017).

Other interventions include interactive activities and discussions that are incorporated into each session which encourages participation and teamwork. This intervention has been shown to improve self-esteem, mental health self-efficacy, and dietary behavior (Tirlea et al., 2016). Other interventions are effective at improving body image of adolescents with dysfunctional eating behavior (Khoshkerdar \& Raeisi, 2020). It does this by raising the awareness that comes when we focus on experiences with a particular topic. It attracts goal-oriented attention (clear attention and focuses on a particular aspect of the experience), focuses on the present (when the mind focuses on the past or future to bring it back to the present), and is non-judgmental (accepts what is happening) (Grossman et al., 2018).

The research conducted shows that changing behavior is proven to help identify appropriate changes in attitude and decision making. The findings of this study emphasize the need for psychosocial support. Working with simple psychological support for the general population can be used to enhance the body image of adolescents (Lee et al., 2017). Good psychological support is expected to improve attitudes and ways of making decisions in determining therapy so that the success of therapy will be more optimal.

\section{CONCLUSION}

There are various interventions capable of providing treatment for body image with varying success. Some psychological interventions are directly (offline) and others are not directly ( online ). Psychological interventions are not directly discussed the use of digital technology intervention in improving body image and a negative into a positive. Interventions in the use of digital technology include the Healthy Eating and Lifestyle Program (HELP) and BodiMojo. This intervention is very easy and efficient to implement today. Also, technology can reduce the cost burden. Indirect psychological intervention with psycho-educational interventions in the form of providing health education, counseling, psychotherapy, and hypnosis greatly helps patients in improving body image in various settings and patient populations. Some of the indirect interventions include Yoga, Mindfulness-Based Stress Reduction (MBSR), and Accuracy and Commitment Therapy (ACT). Comparison of the effectiveness of an intervention must be through testing or research further by clicking to right interventions already exist to be intervention comparators because several factors influence the effectiveness of an intervention, as long intervention, the number of respondents, the involvement of other parties (eg, the age, 


\section{STRADA Jurnal Ilmiah Kesehatan}

DOI: $10.30994 /$ sjik.v9i2.385

ISSN: 2252-3847 (print); 2614-350X (online)

Vol.9 No.2 November 2020 Page.772-789

environment) in giving intervention to respondents, and the age range of respondents. It is necessary to further discuss the effectiveness of direct and indirect interventions for adolescents in Indonesia because the intervention in this Systematic review is mostly carried out outside Indonesia with almost the same characteristics.

\section{REFERENCES}

Barkhordari sharifabad, M., Vaziri-yazdi, S., \& Barkhordari-sharifabad, M. (2020). The effect of teaching puberty health concepts on the basis of a health belief model for improving perceived body image of female adolescents : a quasi-experimental study. BMC Public Health, 20(370), 1-7.

BKKBN. (2017). Survei Demografi dan Kesehatan Indonesia. Retrieved from https://ekoren.bkkbn.go.id/wp-content/uploads/2018/10/Laporan-SDKI-2017-Remaja.pdf

BPS. (2017). Data Sensus. Retrieved from https://jatim.bps.go.id/statictable/2018/02/05/841/persentase-penduduk-usia-15tahun-keatas-di-jawa-timur-menurut-kabupaten-kota-jenis-kelamin-dan-pendidikantertinggi-yang-ditamatkan-2017.html

Cerea, S., Ghisi, M., Bottesi, G., Manoli, T., \& Doron, G. (2020). Cognitive Behavioral Training Using a Mobile Application Reduces Body Image Related Symptoms in High Risk Female University Students: A Randomized Controlled Study. Behavior Therapy, 1-41. https://doi.org/10.1016/j.beth.2020.04.002

Chairani, L.-. (2018). Body Shame dan Gangguan Makan Kajian Meta-Analisis. Buletin Psikologi, 26(1), 12-27. https://doi.org/10.22146/buletinpsikologi.27084

Christie, D., Hudson, L. D., Kinra, S., Chi, I., Wong, K., Nazareth, I., ... Viner, R. M. (2017). A community-based motivational personalised lifestyle intervention to reduce BMI in obese adolescents : results from the Healthy Eating and Lifestyle Programme ( HELP ) randomised controlled trial. BMJ, 102, 695-701. https://doi.org/10.1136/archdischild-2016-311586

Denich, A. U., \& Ifdil. (2015). Konsep Body Image Remaja Putri. Jurnal Konseling Dan Pendidikan, 3(2), 55-61.

Diao, H., Wang, H., Yang, L., \& Li, T. (2020). The impacts of multiple obesity-related interventions on quality of life in children and adolescents : a randomized controlled trial. Health and Quality of Life Outcomes, O(213), 1-10.

Diedrichs, P. C., Atkinson, M. J., Steer, R. J., Garbett, K. M., Rumsey, N., \& Halliwell, E. (2015). Effectiveness of a brief school-based body image intervention 'Dove Confident Me: Single Session' when delivered by teachers and researchers: Results from a cluster randomised controlled trial. Behaviour Research and Therapy, 74, 94104. https://doi.org/10.1016/j.brat.2015.09.004

Dolezal. (2015). The Body and Shame: Phenomenology, Feminism, and the Socially Shaped Body. The United States of America: Lexington Book. Retrieved from http://centreformedicalhumanities.org/ the-body-and-shame-phenomenologyfeminism-and-the-socially-shaped- body-reviewed-by-dr-emily-cock/

Dunstan, C. J., Paxton, S. J., \& McLean, S. A. (2016). An Evaluation of a Body Image Intervention in Adolescent Girls Delivered in Single-sex versus Co-educational Classroom Settings. Eating Behaviors, 1-36. https://doi.org/10.1016/j.eatbeh.2016.03.016

Fogelkvist, M., Aila, S., Kjellin, L., \& Parling, T. (2020). Acceptance and commitment therapy to reduce eating disorder symptoms and body image problems in patients with residual eating disorder symptoms : A randomized controlled trial. Body Image, 


\section{STRADA Jurnal Ilmiah Kesehatan}

DOI: $10.30994 /$ sjik.v9i2.385

ISSN: 2252-3847 (print); 2614-350X (online)

Vol.9 No.2 November 2020 Page.772-789

32, 155-166. https://doi.org/10.1016/j.bodyim.2020.01.002

Georgakaki, S. K., \& Karakasidou, E. (2017). The Effects of Motivational Self-Talk on Competitive Anxiety and Self-Compassion: A Brief Training Program among Competitive Swimmers. Psychology, 08(05), 677-699. https://doi.org/10.4236/psych.2017.85044

Givehki, R., Afshar, H., Goli, F., Scheidt, C. E., Omidi, A., Sciences, M., \& Instructor, F. (2018). Effect of acceptance and commitment therapy on body image flexibility and body awareness in patients with psychosomatic disorders: a randomized clinical trial. Electronic Physician, 10(7), 7008-7016. https://doi.org/http://dx.doi.org/10.19082/7008

Goldfield, G. S., Kenny, G. P., Alberga, A. S., Prud, D., Hospital, R. V., Phillips, P., ... Sigal, R. J. (2015). Effects of Aerobic Training, Resistance Training, or Both on Psychological Health in Adolescents with Obesity: The HEARTY Randomized Controlled Trial. Journal of Consulting and Clinical Psychology, 83(6), 1123-1135.

Grossman, S. L., Campagna, B., Brochu, H., Odermatt, M., \& Annunziato, R. A. (2018). Improving Body Image and Sexual Health Behaviors Among College Women. Journal of American College Health, 1-16. https://doi.org/10.1080/07448481.2018.1454927

Halliwell, E., Dawson, K., \& Burkey, S. (2019). A randomized experimental evaluation of a yoga-based body image intervention. Body Image, 28, 119-127. https://doi.org/10.1016/j.bodyim.2018.12.005

Ishak, S. I. Z. S., Chin, Y. S., Taib, M., Nasir, M., \& Shariff, Z. M. (2016). School-based intervention to prevent overweight and disordered eating in secondary school Malaysian adolescents: a study protocol. BMC Public Health, 16(1101), 1-13. https://doi.org/10.1186/s12889-016-3773-7

Jessie E, M., Ross, K., \& Kevin, T. J. (2011). Attitudinal Assesment of Body Image for Adolescents and Adults. Retrieved from https://psycnet.apa.org/record/2011-20792018

Khoshkerdar, P., \& Raeisi, Z. (2020). The effect of mindfulness-based stress reduction on body image concerns of adolescent girls with dysfunctional eating attitudes. Australian Journal of Psychology, 72, 11-19. https://doi.org/10.1111/ajpy.12265

Krebs, G., Monzani, B., Bowyer, L., Anson, M., Cadman, J., Heyman, I., ... Mataix-cols, D. (2017). ScienceDirect Long-Term Outcomes of Cognitive-Behavioral Therapy for Adolescent Body Dysmorphic Disorder. Behavior Therapy, 48(4), 462-473. https://doi.org/10.1016/j.beth.2017.01.001

Lee, R. L., Leung, C., Chen, H., Louie, L. H. T., Brown, M., Chen, J., ... Lee, P. H. (2017). The Impact of a School-Based Weight Management Program Involving Parents via mHealth for Overweight and Obese Children and Adolescents with Intellectual Disability: A Randomized Controlled Trial. International Journal of Environmental Research and Public Health, 14(1178), 1-18. https://doi.org/10.3390/ijerph14101178

Mandias. (2012). Hubungan Tingkat Pendidikan Dengan Perilaku Masyarakat Desa Dalam Memanfaatkan Fasilitas Kesehatandi Desa Pulisan Kecamatan Likupang Timur Minahasa Utara, 1(1), 45.

Marengo, D., Longobardi, C., Fabris, M. A., \& Settanni, M. (2018). Computers in Human Behavior Highly-visual social media and internalizing symptoms in adolescence: The mediating role of body image concerns. Computers in Human Behavior, 82, 63-69. https://doi.org/10.1016/j.chb.2018.01.003 


\section{STRADA Jurnal Ilmiah Kesehatan}

DOI: $10.30994 /$ sjik.v9i2.385

ISSN: 2252-3847 (print); 2614-350X (online)

Vol.9 No.2 November 2020 Page.772-789

Mumford, \& Choudry. (2000). Body dissatisfaction and eating in slimming and fitness gyms in London and Lahore: A crosscultural study. European Eating Disorders Review, 8, 217-224.

Notoatmodjo. (2010). Promosi Kesehatan Teori Dan Aplikasi Edisi Revisi. Jakarta: Rineka Cipta.

Rodgers, R. F., Donovan, E., Cousineau, T., Yates, K., Mcgowan, K., Cook, E., ... Franko, D. L. (2018). BodiMojo : Efficacy of a Mobile-Based Intervention in Improving Body Image and Self-Compassion among Adolescents. Journal of Youth and Adolescence, 47, 1363-1372. https://doi.org/10.1007/s10964-017-0804-3

Santrock, J. W. (2007). Perkembangan Anak Jilid 1 Edisi Kesebelas. Jakarta: PT. Erlangga.

Seekis, V., Bradley, G. L., \& Duffy, A. (2017). The effectiveness of self-compassion and self-esteem writing tasks in reducing body image concerns, 23, 206-213. https://doi.org/10.1016/j.bodyim.2017.09.003

Seekis, V., Bradley, G. L., \& Duffy, A. L. (2020). Does a Facebook-enhanced Mindful Self-Compassion intervention improve body image? An evaluation study. Body Image, 34, 259-269. https://doi.org/10.1016/j.bodyim.2020.07.006

Stapleton, P., Chatwin, H., Hons, B. A., William, M., Hutton, A., Hons, B., ... Hons, B. A. (2016). EMOTIONAL F REEDOM TECHNIQUES IN THE TREATMENT OF UNHEALTHY EATING BEHAVIORS AND RELATED PSYCHOLOGICAL CONSTRUCTS IN A DOLESCENTS : A RANDOMIZED CONTROLLED PILOT TRIAL. The Journal of Science and Healing, II(II), 1-10. https://doi.org/10.1016/j.explore.2015.12.001

Sundgot-borgen, C., Bratland-sanda, S., Engen, K. M. E., Pettersen, G., Friborg, O., Torstveit, M. K., ... Rosenvinge, J. H. (2018). The Norwegian healthy body image programme : study protocol for a randomized controlled school-based intervention to promote positive body image and prevent disordered eating among Norwegian high school students. BMC Psychology, 6(8), 1-9.

Sundgot-borgen, C., Friborg, O., Kolle, E., Engen, K. M. E., Sundgot-borgen, J., Rosenvinge, J. H., ... Bratland-sanda, S. (2019). The healthy body image ( HBI ) intervention: Effects of a school-based cluster-randomized controlled trial with 12months follow-up. Body Image, 29, 122-131. https://doi.org/10.1016/j.bodyim.2019.03.007

Tanofsky-kraff, M., Shomaker, L. B., Wilfley, D. E., Young, F., Sbrocco, T., Stephens, M., ... Kozlosky, M. (2017). Excess Weight Gain Prevention in Adolescents: Threeyear Outcome following a Randomized-Controlled Trial. J Consult Clin Psychol, 85(3), 218-227. https://doi.org/10.1037/ccp0000153.Excess

Tirlea, L., Truby, H., \& Haines, T. P. (2016). Pragmatic , Randomized Controlled Trials of the Girls on the Go! Program to Improve Self-Esteem in Girls. American Journal of Health Promotion, 30(4), 231-241. https://doi.org/10.1177/0890117116639572

Toole, A. M., \& Craighead, L. W. (2016). Brief self-compassion meditation training for body image distress in young adult women. Body Image, 19, 104-112. https://doi.org/10.1016/j.bodyim.2016.09.001

Trindade, I. A., Marta-Simões, J., Ferreira, C., \& Pinto-Gouveia, J. (2018). Chronic illness-related cognitive fusion explains the impact of body dissatisfaction and shame on depression symptoms in breast cancer patients. Clinical Psychology and Psychotherapy, 25(6), 886-893. https://doi.org/10.1002/cpp.2323

WHO. (2017). Leaving no adolescent behind in health and development in Indonesia. In 5 


\section{STRADA Jurnal Ilmiah Kesehatan}

DOI: $10.30994 /$ sjik.v9i2.385

ISSN: 2252-3847 (print); 2614-350X (online)

Vol.9 No.2 November 2020 Page.772-789

SEPTEMBER 2017.

Wilksch, S. M., Shea, A. O., Taylor, C. B., Wilfley, D., Jacobi, C., \& Wade, T. D. (2018).

Online prevention of disordered eating in at-risk young-adult women: A two-country pragmatic randomised controlled trial Simon, 48(12), 2034-2044. https://doi.org/10.1017/S0033291717003567.Online

Wolfe, W. L., \& Patterson, K. (2017). Comparison of a gratitude-based and cognitive restructuring intervention for body dissatisfaction and dysfunctional eating behavior in college women. Eating Disorders, 1-15. https://doi.org/10.1080/10640266.2017.1279908

Wong, D. L. (2008). Buku Ajar Keperawatan Pediatrik. Jakarta: EGC. 